\title{
A relativistically smeared line profile in the spectrum of the bright Z-source GX $340+0$
}

\author{
Antonino D'Ai* \\ Dipartimento di Scienze Fisiche ed Astronomiche. Università degli Studi di Palermo, Italy \\ E-mail: dai@fisica.unipa.it
}

\section{R.laria}

Dipartimento di Scienze Fisiche ed Astronomiche. Università degli Studi di Palermo, Italy

\section{T.Di Salvo}

Dipartimento di Scienze Fisiche ed Astronomiche. Università degli Studi di Palermo, Italy

\section{G.Matt}

Dipartimento di Fisica, Università degli Studi Roma Tre, Italy.

\section{N.R. Robba}

Dipartimento di Scienze Fisiche ed Astronomiche. Università degli Studi di Palermo, Italy

\begin{abstract}
We present preliminary results of a $50 \mathrm{ks}$ long XMM-Newton observation of the bright Z-source GX 340+0. We study the temporal and spectral variability of the source, performing a time resolved analysis. In the energy spectra, a broad asymmetric emission line in the Fe K $\alpha$ energy band is always present. Its shape is compatible with a relativistically smeared profile arising from reflection on a hot accretion disk extending close to the central accreting neutron star. Despite a significant change in the continuum emission and luminosity, the line profile remains substantially unchanged. The line is produced by recombination of highly ionized iron (Fe XXV), the reflecting disk has an inner radius close to 10 gravitational radii, while the fit requires a high value for the outer disk radius. The inclination of the source is well constrained at $35 \mathrm{deg}$, while the emissivity index is -2.33 .
\end{abstract}

7th INTEGRAL Workshop

September 8-11 2008

Copenhagen, Denmark

\footnotetext{
*Speaker.
} 


\section{Introduction}

GX 340+0 is a Low-Mass X-ray Binary (LMXB) belonging to the class of the Z bright sources [1], and its inferred luminosity is close to the Eddington limit $\left(2 \times 10^{38} \mathrm{erg} / \mathrm{s}\right.$ for a $\left.1.4 \mathrm{M}_{\odot} \mathrm{NS}\right)$.

Temporal analysis studies have shown a complex phenomenology, linked to the accretion state, with characteristics typical of the Z-class [2]. The power spectrum shows a low-frequency (tens of $\mathrm{Hz}$ ) quasi-periodic oscillation when the source resides on its $\mathrm{HB}$ (called Horizontal Branch Oscillation, HBO) while at higher frequencies the source shows, at the same time, twin $\mathrm{kHz}$ QuasiPeriodic Oscillations (kHz QPOs), whose centroid frequencies are correlated with the HBO peak frequency.

The spectral properties of the source have not been fully investigated so far; [3] studied the 2$12 \mathrm{keV}$ spectrum using EXOSAT data; the spectrum could be well described by a single component due to thermal Comptonization of soft photons, emerging from the NS surface, in a hot corona of moderately optical thickness ( $\tau \simeq 5-6$ ); [4] presented the first broadband $(0.1-200 \mathrm{keV})$ spectrum of the source using BeppoSAX data. The spectrum could be decomposed into the sum of a soft thermal component of temperature of $\sim 0.5 \mathrm{keV}$, an optically thick Comptonized component, and an excess at energies above $20 \mathrm{keV}$ that they fitted with a simple power law. A high resolution spectrum of the source was studied by [5], using a Chandra observation; Chandra data clearly showed the presence of an emission line, fitted with a simple Gaussian profile, at $6.57 \mathrm{keV}$ with 40 $\mathrm{eV}$ equivalent width

In this work, we focus on the shape and origin of the reflection features; thanks to the high statistics and good spectral resolution of XMM-Newton, we are able to very well constrain the shape of a broad asymmetric line in the $\mathrm{Fe} \mathrm{K} \alpha$ energy region; we are thus able to determine essential information on the physical configuration of the reflecting medium and to study how the overall X-ray variability influences it.

\section{Data reduction and analysis}

GX 340+0 was observed with XMM-Newton from 2007 September 2 at 13:18:00 to September 3 at 02:32:05 UTC, for a total observing time of $47640 \mathrm{~s}$ (Obs.ID 0505950101). Data were collected by the EPIC-PN instrument Timing Mode. The source region (the background region) has been selected using RAWX $=28-48$ and RAWY $=0-200$ (RAWX $=2-8$ and RAWY $=0-200$ ) columns. No external flaring was present during the observation. We selected only events with PATTERN $\leq 4$ (singles and doubles) and FLAG $=0$ and restricted our analysis to the energy range 2.2-12 $\mathrm{keV}$. We discard PN data below $2.2 \mathrm{keV}$ range and rely on the Reflection Grating Spectrometers (RGS1 and RGS2) data (0.4-2.0 keV range) for the soft energy band, because PN data present strong systematic residuals in this part of the spectrum. PN data have been rebinned in order not to oversample the energy resolution of the instrument by more than a factor of 3, RGS data are rebinned in order to have at least 25 counts per energy channel.

Source, background spectra and response matrices were extracted using the XMM-Newton Science Analysis Software (SAS, ver. 7.1.0). Spectral analysis was performed using Xspec ver. 12.4.0. Temporal analysis was performed using the FTOOLS ver. 6.4.1. 


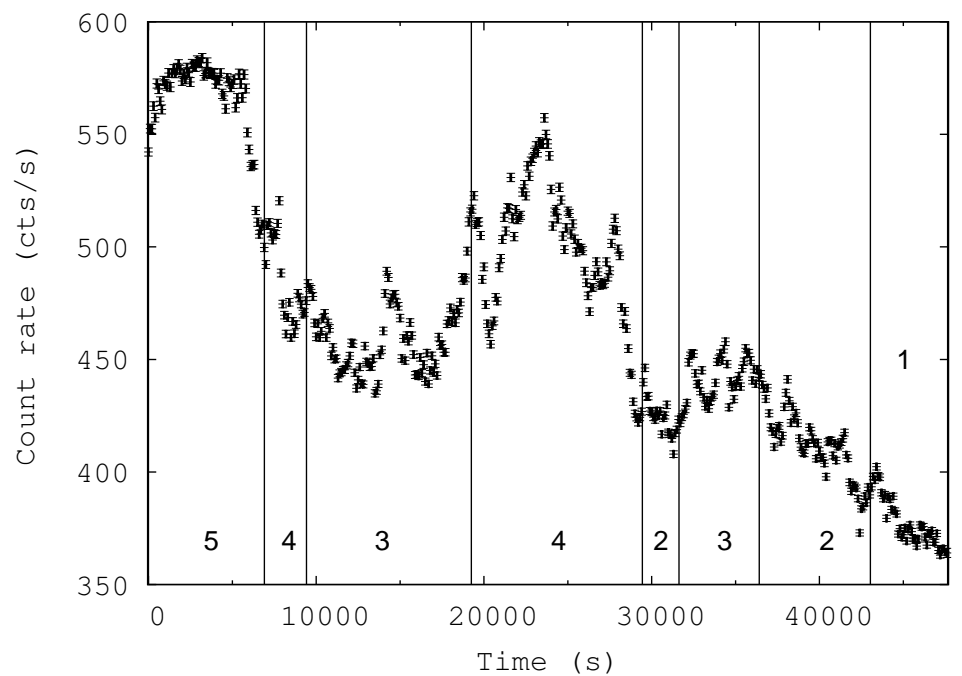

Figure 1 Light curve of GX 340+0 from the EPIC-PN. Bin time $100 \mathrm{~s}$. Vertical lines and numbers inside the boxes indicate the time selection for the corresponding energy spectra.

In Fig.1 we show the light curve (source+background) in the $0.5-10 \mathrm{keV}$ energy range of the entire observation.

Besides the spectral analysis we also performed a study of the temporal variability. We extracted power density spectra (PDS) from the EPIC-PN data in the frequency range $1 / 16 \mathrm{~Hz}$ to 512 Hz. We obtained a single PDS after averaging every 128 PDS, thus having a total of 20 PDS for the entire observation. We fitted the averaged PDSs using two zero-centered Lorentzians, to account for the broadband low frequency noise, a constant term to fit the white noise and a Lorentzian to fit a broad quasi-periodic oscillation. We identify this broad QPO as the HBO [2]; this feature, as clearly shown by [2] is a good tracer of the overall temporal variability, which in turn, can be used to track the accretion state of the source in the X-ray Color-Color Diagram. With the exception of the first two PDS, when the source was at the highest count rate, we clearly detected the HBO in all the remaining PDS (see Fig. 2 and Fig. 3). The HBO centroid span a range of frequencies from 17 to $37 \mathrm{~Hz}$, and we noted this frequency is correlated with the overall X-ray luminosity and count rate, i.e. the higher the QPO frequency, the higher is the X-ray luminosity. We show in Fig.2 the dependence of the HBO peak frequency versus the total source count rate. This relation can be well fitted with a second order polynomial, whose best-fit curve is also shown in the figure. In Fig. 3 we show four representative Lehay normalized power spectra in the 6-100 Hz frequency range. The presence of the $\mathrm{HBO}$ is clerly detected in three of them.

We used these results to derive the good time intervals from which we extracted the corresponding energy spectra. After some trials, we decided to slice the observation into five segments; this choice allows us to trail the spectral evolution of the source, still obtaining a high statistics spectrum to constrain all the spectral parameters. We denote from Spectrum 1 to Spectrum 5 the PN and RGS energy spectra extracted in the time intervals when the HBO frequency was in the $17-22 \mathrm{~Hz}, 22-27 \mathrm{~Hz}, 27-32 \mathrm{~Hz}, 32-37 \mathrm{~Hz},>37 \mathrm{~Hz}$ (or not detected) range, respectively (see the 


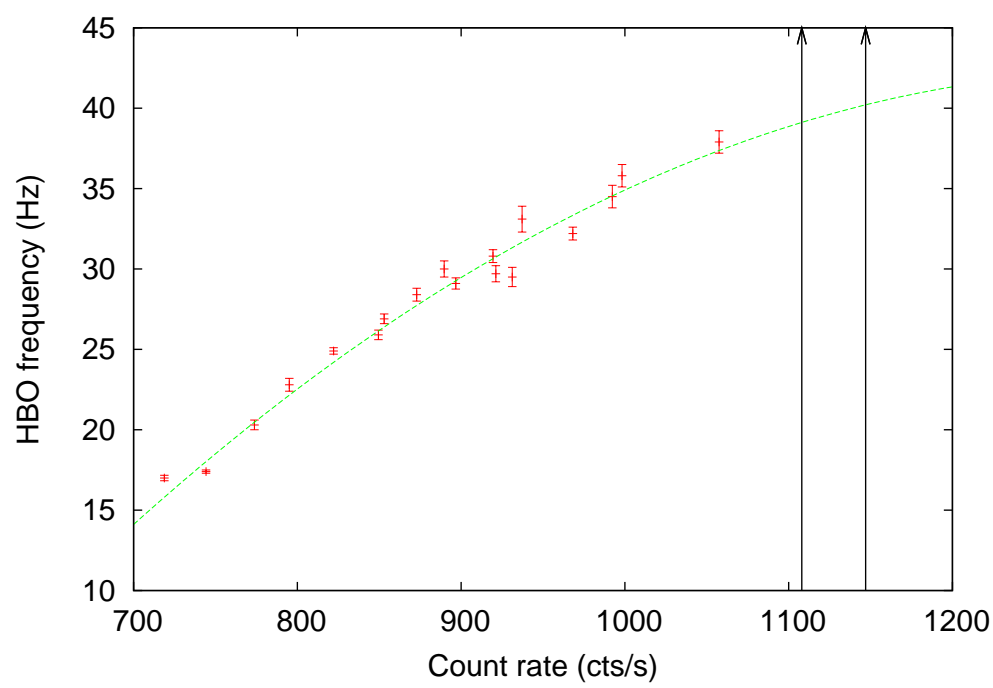

Figure 2 Relation between the centroid frequency of the HBO and the source count rate. The two arrows indicate the average count rate of the time intervals where no HBO is clearly detected. Superimposed to the data, a simple quadratic best-fit of the data is also shown.

time selected intervals shown in Figure 1). ${ }^{1}$

We analyzed the time selected spectra independently, adopting a continuum model consisting of a soft multicoloured thermal disk emission (diskbb component) and a thermal, harder, blackbody emission (component blackbody). We adopted for the interstellar absorption the vphabs model with cross-sections of [6] and table abundances of [7]. We find significant residuals at the neutral iron edge $(7.11 \mathrm{keV})$, leaving the iron abundance free to vary, we find a significant improvement in the $\chi^{2}$ and in the residuals scattering; iron is under-abundant with respect to the assumed [7] interstellar abundance in this direction, as previously noted by [5].

Superimposed to the continuum emission, a few discrete features are always present; a broad emission feature in the $\mathrm{Fe} \mathrm{K} \alpha$ region, a weaker but also broad emission line at $\sim 3.9 \mathrm{keV}$ and an absorption edge, whose energy threshold is in the $8.5-9.0 \mathrm{keV}$ range. The shape of the iron emission line is consistent with a relativistically smeared disk-reflected profile (modelled using the diskline profile of [8]); all the line parameters could be well constrained, except the outer radius for which we derived the largest uncertainties. We fixed this parameter to a reference value of $10^{4}$ $R_{g}$, as any value above 1000 does not alter significantly the $\chi^{2}$ value.

We assume that the $\sim 3.9 \mathrm{keV}$ emission line, that we identify with the Ca XIX Ly $\alpha$ transition is also produced in the disk reflecting plasma, and we fit it using another diskline component, with $\mathrm{R}_{\text {in }}{ }^{2}, \mathrm{R}_{\text {ext }}$, emissivity index and inclination bound to the $\mathrm{Fe} \mathrm{K} \alpha$ diskline component. Best-fitting parameters and associated errors at $90 \%$ c.l. are shown in Table 1.

\footnotetext{
${ }^{1}$ For Spectrum 5, that corresponds to the first $5 \mathrm{ks}$ of the observazion, and to the highest count rate, the spectrum has been extracted using the procedure described in node63 of the SAS v8.0 online documentation, in order to avoid pileup related issues.

${ }^{2}$ Inner and outer disk radii in the diskline model are expressed in units of gravitational radii $\left(R_{g}\right)$, which for a 1.4 solar masses NS corresponds to $2.1 \mathrm{~km}$.
} 

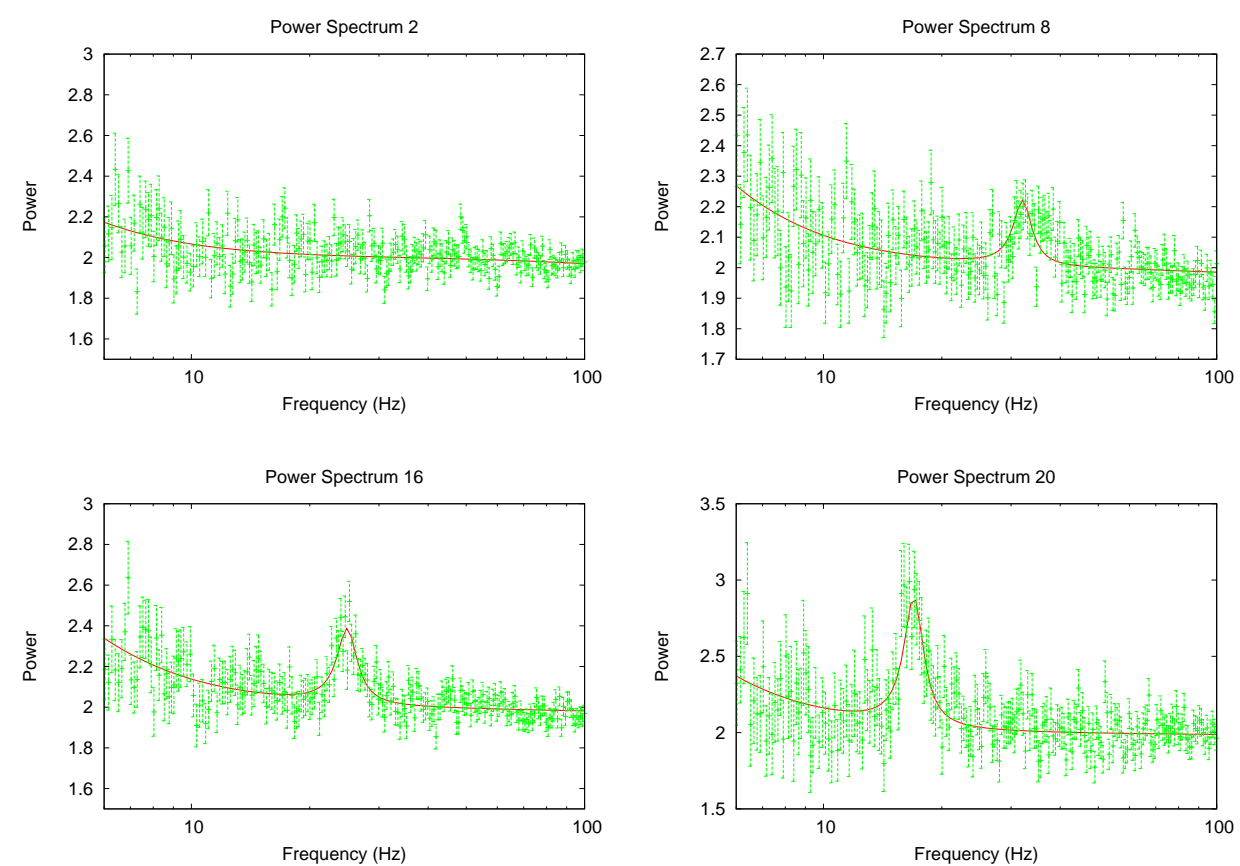

Figure 3 Four representative Lehay normalized power spectra, which show the shift in the frequency position of the HBO during this observation. HBO peak frequencies are undetermined, 32 $\mathrm{Hz}, 25 \mathrm{~Hz}, 17 \mathrm{~Hz}$ for power spectra 2, 8, 16, 20 respectively.
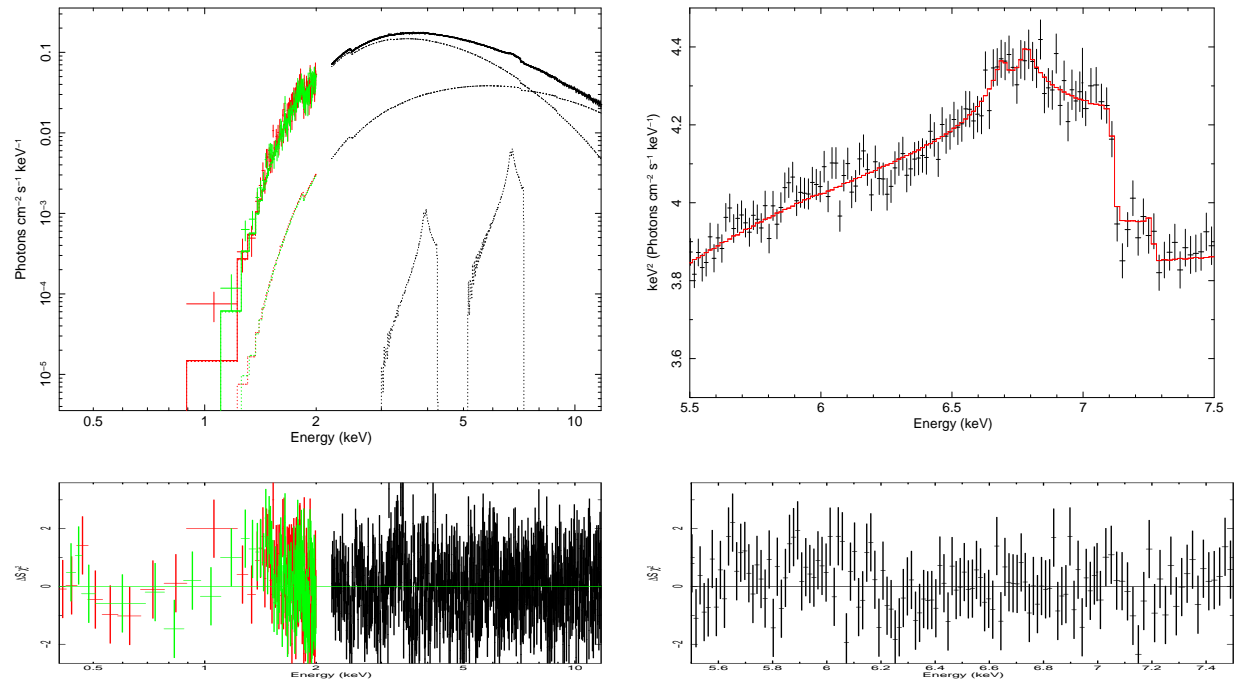

Figure 4 Upper panels: unfolded spectrum (in the $E^{2} \times f(E)$ style), with contribution of the additive components. Lower panels: residuals in units of sigma. Left panels: the $0.4-12 \mathrm{keV}$ energy range, red/green data are RGS1/2 data; black data are PN data. Right panel: the 5.5-7.5 keV region. 
Table 1. Spectral fitting results

\begin{tabular}{|c|c|c|c|c|c|}
\hline & Spectrum 1 & Spectrum 2 & Spectrum 3 & Spectrum 4 & Spectrum 5 \\
\hline HBO freq. (Hz) & $17.7 \pm 0.2$ & $25.7 \pm 0.2$ & $29.4 \pm 0.2$ & $34.6 \pm 0.4$ & $37-40^{\mathrm{A}}$ \\
\hline $0.5-2.0 \mathrm{keV}$ Flux $^{\mathrm{B}}$ & 2.69 & 2.99 & 3.22 & 3.55 & 4.17 \\
\hline 2.0-10.0 keV Flux ${ }^{\mathrm{B}}$ & 9.38 & 10.45 & 11.51 & 12.77 & 14.82 \\
\hline$N_{\mathrm{H}}^{\mathrm{C}}$ & $9.42_{-0.21}^{+0.26}$ & $9.84 \pm 0.20$ & $9.89 \pm 0.19$ & $10.23 \pm 0.14$ & $10.51 \pm 0.27$ \\
\hline Iron Abundance & $0.84_{-0.2}^{+0.13}$ & $0.75 \pm 0.11$ & $0.64 \pm 0.07$ & $0.72 \pm 0.08$ & $0.68 \pm 0.13$ \\
\hline$k T_{\text {disk }}(\mathrm{keV})$ & $1.60_{-0.11}^{+0.23}$ & $1.85_{-0.10}^{+0.09}$ & $1.88 \pm 0.08$ & $2.06_{-0.02}^{+0.03}$ & $2.07 \pm 0.3$ \\
\hline $\mathrm{R}_{D B B} \mathrm{P}(\mathrm{km})$ & $9.7_{-1.7}^{-1.1 .3}$ & $7.8 \pm 0.6$ & $7.6 \pm 0.4$ & $\begin{array}{r}-0.02 \\
8.0 \pm 0.4\end{array}$ & $10.5 \pm 0.4$ \\
\hline$k T_{b b}(\mathrm{keV})$ & $2.55_{-0.12}^{+0.22}$ & $2.89_{-0.20}^{+0.23}$ & $2.98 \pm 0.20$ & $>3.5$ & $>3.5$ \\
\hline $\mathrm{Fe} \mathrm{E}_{\text {line }}(\mathrm{keV})$ & $6.70 \pm 0.03$ & $6.72 \pm 0.03$ & $6.67 \pm 0.03$ & $6.72 \pm 0.03$ & $6.81 \pm 0.14$ \\
\hline $\mathrm{R}_{\text {in }}\left(\mathrm{R}_{g}\right)$ & $16_{-8}^{+20}$ & $13_{-7}^{+5}$ & $14 \pm 2$ & $13_{-7}^{+6}$ & $7_{-1}^{+18}$ \\
\hline $\mathrm{R}_{\text {ext }}\left(\mathrm{R}_{g}\right)$ & $10^{4}$ & $10^{4}$ & $10^{4}$ & $10^{4}$ & $10^{4}$ \\
\hline Incli. (deg) & $40_{-5}^{+10}$ & $33_{-2.0}^{+2.5}$ & $36.2_{-1.3}^{+0.9}$ & $35.7 \pm 1.7$ & $30_{-14}^{+6}$ \\
\hline Betor $^{\mathrm{F}}$ & $2.38_{-0.10}^{+0.14}$ & $2.46_{-0.07}^{+0.11}$ & $2.52 \pm 0.07$ & $2.47 \pm 0.07$ & $2.66_{-0.2}^{+0.3}$ \\
\hline Fe Norm. ${ }^{\mathrm{G}}$ & $5.5_{-0.8}^{-0.10}$ & $5.1 \pm 1.0$ & $5.7 \pm 0.5$ & $4.0 \pm 0.6$ & $5.6_{-1.2}^{+3.2}$ \\
\hline Line Equivalent Width (eV) & 60 & 43 & 43 & 33 & 33 \\
\hline $\mathrm{Ca} \mathrm{E}_{\text {line }}(\mathrm{keV})$ & $3.87 \pm 0.07$ & $3.93_{-0.10}^{+0.17}$ & $3.94 \pm 0.05$ & $3.94 \pm 0.06$ & $3.92 \pm 0.07$ \\
\hline Ca Norm. ${ }^{G}$ & $0.55_{-0.06}^{+0.06}$ & $0.63_{-0.1}^{+0.10}$ & $1.5 \pm 0.3$ & $1.4_{-0.9}^{+1.9}$ & $3.7_{-1.4}^{+2.7}$ \\
\hline Line Equivalent Width (eV) & 4.2 & 2.2 & 3.0 & 4.1 & 6.0 \\
\hline Edge $\mathrm{E}(\mathrm{keV})$ & $8.85 \pm 0.10$ & $8.97 \pm 0.10$ & $8.94 \pm 0.10$ & $8.80_{-0.07}^{+0.10}$ & $8.91_{-0.09}^{+0.22}$ \\
\hline Edge $\tau\left(\times 10^{-2}\right)$ & $4 \pm 1$ & $3 \pm 1$ & $3 \pm 1$ & $3.0_{-0.7}^{+0.06}$ & $\begin{array}{r}-0.09 \\
3.7 \pm 1.6\end{array}$ \\
\hline$\chi_{\text {red }}^{2}(d o f)$ & 0.974 (674) & $1.083(771)$ & $1.244(908)$ & $1.186(866)$ & $1.084(663)$ \\
\hline
\end{tabular}

${ }^{\mathrm{A}} \mathrm{QPO}$ not resolved in the PSD, value inferred to be in this range from [2]. See also Fig.2

${ }^{\mathrm{B}}$ Unabsorbed flux in units $10^{-9} \mathrm{ergs}^{-2} \mathrm{~s}^{-1}$.

CIn units of $10^{22}$ atoms $\mathrm{cm}^{-2}$.

${ }^{\mathrm{D}}$ Inner disk radius in $\mathrm{km}$, as derived from the normalization parameter $N$ of the diskbb component: $R_{D B B}=D \sqrt{N / \cos (\theta)}$; for the calculation we assume a distance $(D)$ of $11 \mathrm{kpc}$ and an inclination angle $(\theta)$ of $36.7 \mathrm{deg}$.

${ }^{\mathrm{E}}$ Frozen parameter during the fitting procedure.

${ }^{\mathrm{F}}$ Index of the emissivity power law, that scales as $\mathrm{r}^{- \text {(Betor) }}$.

${ }^{\mathrm{G}}$ Normalization values of the diskline component in units of $10^{-3}$ photons $/ \mathrm{cm}^{-2} / \mathrm{s}$.

Note. - Best-fitting values and associated errors for spectra 1-5. Errors quoted at $\Delta \chi^{2}=2.7$. 


\section{Discussion}

During this XMM-Newton observation we can closely follow the spectral and temporal evolution of GX $340+0$ in its HB. We used the HBO peak frequency as tracer of the overall X-ray variability. At the leftmost point of the $\mathrm{HB}$, corresponding to the lowest count rate and to a frequency of the $\mathrm{HBO}$ of $\sim 17 \mathrm{~Hz}$, we derive the lowest temperatures both for the disk (1.61 keV) and the hard emission $(2.55 \mathrm{keV})$; as the source moves towards the hard apex, there is a continuum rising of the temperatures and of the X-ray flux; assuming a distance of $11 \pm 3 \mathrm{kpc}$ and an inclination of the disk as inferred from the diskline profile, we derive a change in the source luminosity from $(1.67 \pm 0.1) \times 10^{38} \mathrm{erg} / \mathrm{s}$ (spectrum 1) to $(2.57 \pm 0.2) \times 10^{38} \mathrm{erg} / \mathrm{s}$ (spectrum 5). The inner disk radius as derived from the diskbb normalization (see Table 1) gives a value in the 7-11 km range; considering that the inner disk radius can be underestimated by a factor of two [9], we find that these values are in agreement with the ones derived from the diskline line profile. Despite this remarkable continuum change, the profile of the broad iron diskline does not sensibly change. The rest frame energy of the diskline component present in the iron region indicates that the line is most probably produced by He-like iron ions at a short distance from the compact source. The equivalent width of the line decreases from spectrum $1(\sim 60 \mathrm{eV})$ to spectrum $5(\sim 30 \mathrm{eV})$, indicating that the line does not respond to the soft X-ray flux, which, on the contrary increases, but presumably to the hard X-ray emission, above $12 \mathrm{keV}$, which falls out from the observed spectral coverage of the EPIC/PN instrument. The inclination angle of the disk and the emissivity index are very well constrained by our fits, with relative uncertainties of only a few percent. Contrary to what found in galactic black holes, the emissivity index is not very steep, indicating a more extended illuminating corona above the disk [8].

The smooth change in the continuum parameters and the similar values obtained for the emissivity index, inclination and inner radius of the diskline led us to more closely inspect the 6.4-7.0 keV Fe K $\alpha$ region. In this energy range we found no complex continuum curvatures due to the changing spectral states and a general consistency of the line shape. We summed, thereafter, the spectra in order to increase the statistics and to better constrain the line parameters under the motivated assumption that its parameter variations are beneath the statistical resolving power of the EPIC/PN-data. We adopted the same continuum model as previously described, finding, as expected, that the best-fitting values of the continuum are a weighted sum of the best-fitting values shown in Table 1. We obtained for the averaged diskline parameters: a rest frame energy of the line $6.71_{-0.02}^{+0.1} \mathrm{keV}$, inner radius $\mathrm{R}_{i n}=12.8_{-6}^{+9} R_{g}$, outer radius $\mathrm{R}_{\text {ext }}=4400_{-2600}^{+3800} R_{g}$, inclination angle $34.8_{-1.6}^{+1.4} \mathrm{deg}$ and emissivity index $2.33_{-0.20}^{+0.17}$.

Besides the broad emission iron line, we found evidence in our spectra of two other reflection signatures: an emission line at $\sim 3.94 \mathrm{keV}$, whose equivalent width is some $\mathrm{eV}$, and an absorption edge in the 8.8-9.0 keV range. The former could be due to the resonant transition of Ca XIX (see also [10] for similarities), while the edge can be related to Fe XXV absorption, which is also expected from the presence of the emission line and the inclination angle of the system.

Most theoretical models on the QPO generation in NS LMXB agree to identify the shortest dynamical timescales, i.e. the highest frequency QPO at $\sim 1 \mathrm{kHz}$, with the frequency of the Keplerian motion of matter at the inner rim of an accretion disk. From the HBO frequency and the relation known to exist between this and the upper $\mathrm{kHz}$ QPO frequency [2], we can infer the up- 
per $\mathrm{kHz}$ QPO to be in the $550-750 \mathrm{~Hz}$ frequency range. For a typical $1.4 \mathrm{M}_{\odot} \mathrm{NS}$ this frequency range corresponds to an inner disk radius of $12.1-9.8 \mathrm{R}_{\mathrm{g}}$. The diskline inferred inner radii for the time-selected spectra spectra are all fully consistent within this range, and give support to the identification of highest QPO frequency with the inner disk Keplerian frequency. Our fit results also seem to indicate a decrease in the value of the inner disk radius as the source moves towards higher luminosities. The errors associated to this parameter are, however, still too large to claim a strict correlation and longer exposures are needed to prove the significance of such a relation.

Acknowledgments

Authors wish to express their gratitude to the EPIC/PN team and, especially, to Dr. Matteo Guainazzi for the kind assistance offered us in the process of data extraction and reduction of the PN data.

\section{References}

[1] Hasinger, G., \& van der Klis, M. 1989, A\&A, 225, 79

[2] Jonker, P. G., et al. 2000, ApJ, 537, 374

[3] Schulz, N. S., \& Wijers, R. A. M. J. 1993, A\&A, 273, 123

[4] Lavagetto, G., Iaria, R., di Salvo, T., Burderi, L., Robba, N. R., Frontera, F., \& Stella, L. 2004, Nuclear Physics B Proceedings Supplements, 132, 616

[5] Ueda, Y., Mitsuda, K., Murakami, H., \& Matsushita, K. 2005, ApJ, 620, 274

[6] Verner, D. A., Ferland, G. J., Korista, K. T., \& Yakovlev, D. G. 1996, ApJ, 465, 487

[7] Wilms, J., Allen, A., \& McCray, R. 2000, ApJ, 542, 914

[8] Fabian, A. C., Rees, M. J., Stella, L., \& White, N. E. 1989, MNRAS, 238, 729

[9] Merloni, A., Fabian, A. C., \& Ross, R. R. 2000, MNRAS, 313, 193

[10] D’Aí, A.,Iaria, R., Di Salvo, T., Lavagetto, G.,\& Robba, N. R. 2007, ApJ, 671, 2006 